\title{
Nail Ridging, CTCAE
}

National Cancer Institute

\section{Source}

National Cancer Institute. Nail Ridging, CT CAE. NCI Thesaurus. Code C143697.

A disorder characterized by vertical or horizontal ridges on the nails. 